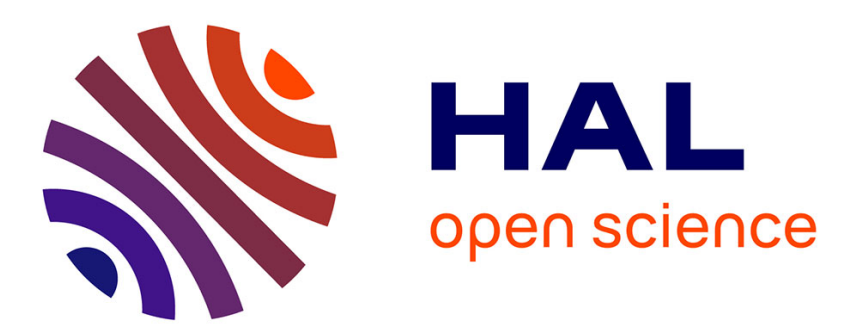

\title{
Temporal processing in the striatum: interplay between midbrain dopamine neurons and striatal cholinergic interneurons
}

Anne-Caroline Martel, Paul Apicella

\section{- To cite this version:}

Anne-Caroline Martel, Paul Apicella. Temporal processing in the striatum: interplay between midbrain dopamine neurons and striatal cholinergic interneurons. European Journal of Neuroscience, 2020. hal-03033201

\author{
HAL Id: hal-03033201 \\ https://hal.science/hal-03033201
}

Submitted on 1 Dec 2020

HAL is a multi-disciplinary open access archive for the deposit and dissemination of scientific research documents, whether they are published or not. The documents may come from teaching and research institutions in France or abroad, or from public or private research centers.
L'archive ouverte pluridisciplinaire HAL, est destinée au dépôt et à la diffusion de documents scientifiques de niveau recherche, publiés ou non, émanant des établissements d'enseignement et de recherche français ou étrangers, des laboratoires publics ou privés. 


\title{
Temporal processing in the striatum: interplay between midbrain dopamine neurons and striatal cholinergic interneurons
}

\author{
Anne-Caroline Martel and Paul Apicella \\ Institut de Neurosciences de la Timone UMR 7289, Aix Marseille Université, CNRS, \\ 13385, Marseille, FRANCE
}

$\begin{array}{ll}\text { Corresponding author : } & \text { Paul APICELLA } \\ & \text { Institut de Neurosciences de la Timone UMR } 7289 \\ & \text { Aix Marseille Université, CNRS } \\ & \text { 13385 Marseille FRANCE } \\ & \text { paul.apicella@univ-amu.fr }\end{array}$

Running title: dopaminergic and cholinergic control of timing

Number of pages : 23

Number of figures : 3

Number of words for Abstract : 241

Number of words for the whole manuscript (including references and figure legends): 6218

Keywords : basal ganglia, interval timing, neuronal recording, rodent, non-human primate

List of abbreviations:

DA: dopamine

ChI: cholinergic interneuron

TAN: tonically active neuron

MSN: medium spiny neuron

SBF: striatal beat frequency 


\begin{abstract}
There has been considerable progress in recent years towards understanding the neuronal mechanisms mediating time perception. Notably, the striatum and its dopamine (DA) input from the ventral midbrain are considered to be central for timing on the scale of hundreds of milliseconds and seconds. The cholinergic interneurons (ChIs) of the striatum provide an extensive local innervation which closely interacts with striatal DA afferents. Both neuronal systems have been shown to influence synaptic plasticity to shape the transfer of information through the striatum. Given their cooperative role in regulating striatal output pathways, DA and cholinergic inputs may have distinct but complementary roles in timing processes. Electrophysiological recordings from behaving animals have provided evidence that responses of midbrain DA neurons and striatal tonically active neurons (TANs), presumed ChIs, to motivationally relevant events are sensitive to the predicted time of these events. Namely, changes in neuronal activity are reduced or absent at times when events are more expected, indicating that temporal aspects of prediction play an important role in the responsiveness of these two neuronal systems. Recently, new findings have further suggested that DA neurons and cholinergic TANs are both involved in the ability to keep track of elapsed time. These two systems appear to work in parallel in initiating the timing process at the beginning of an interval to be timed. It therefore appears that DA and ChI signaling could participate in striatal processing that is crucial for the control of timing behavior.
\end{abstract}




\section{Introduction}

Information about timing is encoded in a distributed network of brain regions including the basal ganglia, cerebellum and multiple areas of the frontal and parietal cortices (Buhusi and Meck, 2005; Coull et al., 2011; Merchant et al., 2013). Numerous lines of evidence, from human neuroimaging studies to inactivation experiments in animals, suggest that the striatum, the main recipient of afferents to the basal ganglia, plays a central role in interval timing, defined as the perception of durations in the range of hundreds of milliseconds to several seconds. The main population of neurons in the striatum, the GABAergic projection neurons, also called medium spiny neurons (MSNs), has been at the center of research on how striatal circuits could convey information about the passage of time. Previous electrophysiological studies reporting neuronal correlates of timing in the striatum have focused on MSNs in rodents (Matell et al., 2003; Mello et al., 2015; Gouvêa et al., 2015; Bakhurin et al., 2017) and monkeys (Jin et al., 2009; Chiba et al., 2015; Wang et al., 2018), whereas the role of local interneuron circuits that exert influence on striatal function has not been considered. In particular, cholinergic interneurons (ChIs) have long been known to be important, along with DA input, for various forms of corticostriatal synaptic plasticity (Di Filippo et al., 2009) and they play a powerful role in modulating DA release in the striatum (Cragg, 2006; Threlfell et al., 2012; Cachope \& Cheer, 2014). Although they represent only $\sim 3 \%$ of the total neuronal population in the striatum, ChIs give rise to extensive axonal arbors that exert an important regulation on striatal output in cooperation with DA (Calabresi et al., 2000; Pisani et al., 2007). Even though theoretical models of interval timing have tried to specify a role for the striatum and its DA input in the control of timing behavior, they do not outline how ChIs contribute to the neuronal mechanisms that underlie timing functions. The present review summarizes the relevant data linking time processing with DA and cholinergic transmissions in the striatum. We particularly 
focus on recent neuron recording studies from midbrain DA neurons and striatal ChIs that seek to clarify how these two neuronal systems contribute to timing processes.

\section{Involvement of striatal dopamine transmission in temporal processing}

Numerous studies have suggested that timing mechanisms depend on the integrity of midbrain DA neurons, particularly those projecting from the substantia nigra pars compacta to the dorsal striatum (Meck, 1983; 1996). Abnormal temporal processing has been reported in pathological conditions associated with DA dysfunction, such as Parkinson's disease (Pastor et al., 1992; Parker et al., 2013; Merchant et al., 2008; Mioni et al., 2018), schizophrenia (Rammsayer, 1990; Volz et al., 2001; Davalos et al., 2003; Hwang et al., 2010), and attention deficit disorder (Yang et al., 2007; Hwang et al., 2010; Smith et al., 2013). Pharmacological studies in both animals and humans and lesion studies in animals have extensively documented the link between DA and temporal processing (Meck, 1996). For example, impaired timing variability has been recently reported after the systemic injection of the dopamine D2 receptor agonist quinpirole in monkeys trained to produce rhythmic movements (Yc et al., 2019). In addition, there is evidence for impaired timing behavior after specific inactivation of substantia nigra DA neurons, using optogenetic and chemogenetic approaches in mice trained to discriminate the duration of time intervals (Soares et al., 2016). Also, a neuroimaging study in human subjects performing a timing task has shown that a pharmacologically-induced reduction of DA levels can decrease the time-dependent activation within the striatum, in parallel with disturbances in timing ability (Coull et al., 2012).

The most widely accepted explanation about how DA activity intervenes in temporal processing is that striatal DA levels modulate the speed of a putative internal clock supposedly designed to produce a representation of elapsed time (Merchant et al., 2013; Paton \& Buonomano, 2018). According to this view, decreased striatal DA transmission, in response to 
pathological or experimental situations, is associated with a reduction in clock speed (i.e., a slowing of temporal processing) leading to an overestimation of time, and the reverse occurs in case of increased DA transmission associated with underestimation (Meck, 1983; 1996). Although this internal clock model explains a number of behavioral results obtained in a variety of timing tasks, in both animals and humans, the underlying neurobiological mechanisms were not specifically addressed. A more elaborate view has been proposed to connect theoretical accounts of temporal processing with possible neuronal substrates. The so-called striatal beat frequency (SBF) model of Matell and Meck (Matell \& Meck, 2000; Matell et al., 2004) highlights the relationship between corticostriatal circuits and midbrain DA neurons. The model assumes that detection of coincident cortical activity by MSNs may encode the representation of elapsed time. The start signal to begin timing is considered to be marked by a phasic activation of DA neurons, acquired through learning, that synchronizes the oscillations of cortical neurons at the onset of the interval to be timed. The SBF model mainly focuses on the influence of cortical neuronal oscillators on MSNs located in the dorsal striatum under the influence of DA signals. However, while the idea is interesting, there is little physiological evidence supporting such a relation between MSN activity and cortical oscillatory activity and the model thus remains largely speculative. In sum, the above models of timing propose a central role for the DA transmission, with an influence that arises at different stages of time processing according to the model. The most popular theory (clock model) considers that the level of DA modulates the speed of an internal time keeping mechanism, whereas another theoretical perspective (SBF model) assumes that the DA modulation serves primarily as a start signal to keep track of elapsed time. Despite the close interplay and dependence between the two neuronal systems in the striatum, it is noteworthy that both theoretical models focus exclusively on the implication of DA input and do not account for a specific role of striatal cholinergic transmission in time processing. 
A more recent theoretical view considers that striatal encoding of elapsed time relies on dynamic changes in the population of MSNs, a model referred to as « population clocks » (Buonomano \& Karmarkar, 2002). Although there is a growing body of experimental evidence in favor of such a population coding (Gouvêa et al., 2015; Mello et al., 2015; Bakhurin et al., 2017; Wang et al., 2018), the link between DA and cholinergic modulations and neuronal dynamics in striatal output circuits has yet to be clarified.

\section{Involvement of striatal cholinergic transmission in temporal processing}

Compared to DA, far less is known about the role that cholinergic transmission exerts on the encoding of time. Pharmacological manipulations by systemic injections of cholinergic drugs in rodents have established a link between cholinergic activity and timing behavior (Meck, 1983; 1996; 2002; Meck \& Church, 1987). Within the framework of the internal clock model and based on the dynamics of the observed effects on timing behavior, it has been suggested that cholinergic manipulations impair timing by disturbing storage of temporal information into memory. These data led to the proposal that DA neurons of the substantia nigra projecting to the dorsal striatum are involved in temporal processing speed, whereas cholinergic neurons of the basal forebrain projecting to the frontal cortex are thought to be important for the temporal memory representation (Meck, 1996; Meck \& Church, 1987; Coull et al., 2011; Oprisan \& Buhusi, 2011). However, systemic pharmacological injections are unspecific and do not allow to determine the precise contribution of the intrinsic cholinergic innervation of the striatum, thus requiring a more selective approach by using local microinjections. Some studies have used local application of cholinergic receptor antagonists into the striatum (Ztaou et al., 2016) or targeted inactivation of ChIs over different regions of the striatum (Okada et al., 2014; Aoki et al., 2015; 2018), but they did not address the issue of the role of ChIs in timing processes; rather there is a current, growing interest in the role of these interneurons in the control of 
flexible switching of behaviors (Brown et al., 2010; Prado et al., 2017; Bell et al., 2019) and monitoring of current environmental state during behavior (Apicella, 2007; Bradfield et al., 2013; Stalnaker et al., 2016), leaving aside the question of their role in time processing. Interestingly, disturbances in timing behavior have been reported in patients with Tourette syndrome (Vicario et al., 2010), a neuropsychiatric disorder in which the density of specific types of striatal interneurons, including ChIs, has been shown to be decreased (Kataoka et al., 2010).

\section{Single neuron recordings in behaving animals}

More direct evidence in support for the contribution of midbrain DA neurons and striatal ChIs to the encoding of time has come from electrophysiological studies in which animals were exposed to variation in the timing of rewarding events. Studies in monkeys have demonstrated that receipt of a reward at a fully expected time elicits little or no change in the activity of DA neurons, whereas a brief increase in firing rate emerges when the timing of reward becomes less predictable (Hollerman \& Schultz, 1998; Fiorillo et al., 2008; Kobayashi \& Schultz, 2008). This is illustrated in Fig.1A in which reward delivered earlier or later than its usual time led to a strong DA response. Other studies have reported that DA responses are reduced as the occurrence of a behaviorally relevant stimulus becomes increasingly predicted over time, a phenomenon referred to as the hazard rate (Fiorillo et al., 2008; Pasquereau \& Turner, 2015). As a whole, these data indicate that the strength of DA responses is shaped by the degree of temporal predictability of rewarding events.

Most of the ideas about the role of the intrinsic striatal cholinergic innervation come from recordings of tonically active neurons (TANs) that are presumed to be ChIs. TANs can be distinguished from other striatal neurons based on distinguishable electrophysiological features and changes in activity in response to rewarding events (Apicella, 2002). Whether there are 
other striatal interneurons than ChIs among the group identified as TANs remains a possibility given limitations of current electrophysiological recording techniques in behaving monkeys to probe the identity of sampled neurons (Apicella, 2017). As for DA neurons, TANs participate in signaling stimuli predictive of reward and the reward itself. An important property of TAN responses is their dependency on the temporal predictability of rewarding events. As reported in our earlier studies, TAN responses to reward, consisting mainly of brief decreases in firing rate (the so-called "pause"), were reduced or even abolished when the timing of reward can be predicted in Pavlovian and instrumental tasks (Apicella et al., 1997; 2006; Ravel et al., 2001). As can be seen in the example neuron shown in Fig.1B, the TAN response to reward increased with time intervals shorter or longer than the habitual one, in a manner quite similar to what has been described for DA neurons. Similarly, in an arm reaching task, the time delay between an instruction cue and the subsequent movement-triggering stimulus is a major determinant of TAN responses, with lengthening the delay having an increasing effect on the response to the trigger and a decreasing effect on the response to the cue (Sardo et al., 2000). It is therefore evident from this brief overview of their response properties that DA neurons and ChIs are both sensitive to temporal aspects of stimulus prediction. However, we lack information about their precise contribution to the striatal mechanisms mediating temporal information processing.

\section{The role of midbrain dopamine neurons and striatal cholinergic TANs in the processing of temporal information}

In the research reviewed above, DA and ChI systems were studied in the context of tasks that required animals to use temporal regularities in sequential events to predict the onset of a reward-predicting stimulus or the reward itself. In such conditions, the representation of elapsed time could be assumed to be "implicit", as it relies upon predictive temporal relations between events acquired through extensive practice. There are very few studies that have evaluated the 
contribution of DA neurons and striatal ChIs to timing under conditions that require an overt estimate of elapsing time to guide behavior, thus focusing on "explicit" timing mechanisms rather than "implicit" ones (Coull \& Nobre, 2008; Coull et al., 2011).

Only one study has investigated DA activity in a condition in which animals were required to produce an overt estimate of elapsed time (Soares et al., 2016). The researchers trained mice to categorize the durations of intertone intervals as shorter or longer than a prelearned duration (Fig.2A). During this temporal discrimination task, DA activity in the substantia nigra pars compacta was measured using fiber photometry-based calcium recording. As can be seen in Fig.2B, DA activity increased after the first and the second tone defining the time interval and this increase was stronger when the interval was judged as shorter, reflecting an underestimation of the duration of the interval. Conversely, a weaker DA increase was observed when the interval was judged as longer (i.e., overestimation). This suggests that higher or lower level of DA activity was closely related to the animals' estimates of the duration of time intervals (Fig.2C). In the same study, the authors demonstrated that the observed changes in DA activity are functionally relevant because optogenetic manipulations of nigral DA signaling affect timing performance. This is the first study to demonstrate a link between DA activity with the animal's ability to react in a temporally controlled manner.

As regards striatal ChIs, a number of recording studies have reported changes in TAN activity that are dependent on temporal prediction, but in none of them were animals required to use temporal information to guide behavior. Recently, we have recorded from single TANs in the striatum of two rhesus monkeys trained to perform a timing task in which they had to initiate self-timed reaching movements after a specified time interval has passed (Martel \& Apicella, unpublished results). Animals were required to produce an appropriate time interval that was close to a pre-learned minimum waiting period before initiating an action (Fig.3A left part), a performance that relies on internally scaled estimates of duration. We studied 200 TANs 
in this duration estimation task using recording methods described in detail previously (e.g., Apicella et al., 2011). About one third of the recorded TANs (73 of 200 neurons, 37\%) displayed significant changes in activity in response to the cue that indicates the beginning of the time interval, essentially consisting of a decrease in firing rate. This pause response was similar in magnitude regardless of whether the cue was associated with an ensuing short or long interval (Fig.3B). Also, we found no evidence that the TAN response to the cue might reflect the monkey's accuracy of estimating interval duration (Fig.3C). Although studies in rodents and monkeys are difficult to compare because of differences in task design, the lack of sensitivity of TANs to timing accuracy appears to contrast with the level of DA activity which is predictive of performance during time-guided behavior (Soares et al., 2016).

In the same monkeys, we also tested the effects of interval durations when animals were passively exposed to a visual stimulus followed by the delivery of reward in a Pavlovian conditioning task (Fig. 3A right part). This design allowed us to look specifically at the influence of a stimulus providing advance information about the timing of reward in a condition that excludes possible confounding factors that were linked to target reaching. A total of 90 TANs were recorded in the Pavlovian protocol and 38 of them (42\%) responded to the stimulus that serves as a timing cue. At the population level, the TAN pause response (Fig.3D) was essentially similar to that observed in the duration estimation task. However, by focusing on a subset of 31 TANs that were tested in both testing conditions, we found that subsets of TANs responded to the timing cue selectively in one condition, either duration estimation task or Pavlovian protocol (Fig.3E). That a substantial fraction of TANs responded only in one testing condition supports the hypothesis that responses do not strictly follow timing cues themselves, but rather do so according to the contextual demands of the task. Such a context dependency in TAN activity is a recurring topic of discussion in the literature on the role of the ChIs (Apicella, 2007; Bradfield et al., 2013; Stalnaker et al., 2016). Our results are consistent with the idea that 
the cholinergic TAN system might support recognition of previously learned temporal contexts. However, although changes in TAN activity have been hypothesized to be under the control of context, the mechanisms through which these interneurons use context detection to regulate striatal output and potentially influence timing behavior are far from being understood. In particular, there are difficulties in most neurophysiological studies to isolate the encoding and processing of temporal information from the detection of the context. To better test whether TANs preferentially encode the context or time, it would be conceivable to use a task design in which precise timing of actions or rewarding events can occur in different contexts. For example, one stimulus (temporal cue) instructs the animal about the duration of the delay prior to movement, while another stimulus (contextual cue) indicates that the animal must make or withhold movement to obtain a reward (a condition that could be referred to as a "timedependent Go-NoGo task"). Using trial blocks in which the delay time and reward contingency are independently varied would make it possible to compare the effects of each task variable on TAN activity.

The contribution of dopamine-acetylcholine interactions to temporal processing in the striatum

As mentioned in this review, there is experimental evidence from pharmacological and lesion studies indicating that manipulations of DA and cholinergic transmissions cause disturbances in timing behavior. It is mainly on the basis of these results that it has been proposed that DA mediates the speed of an internal clock, whereas acetylcholine would be rather involved in encoding temporal memory (Meck, 1996; Buhusi \& Meck, 2005) or in detecting a learned context (Apicella, 2007; Bradfield et al., 2013; Stalnaker et al., 2016). On the other hand, a more direct assessment of the activity of DA neurons (Soares et al., 2016) and ChIs during 
timing tasks suggests that these two neuronal systems appear to work in parallel in initiating the timing process at the beginning of an interval to be timed. In this regard, midbrain DA neurons and striatal ChIs may provide a signal potentially suitable for time processing in the striatum. However, unraveling their differential sensitivity across studies is difficult because of variations of experimental designs, notably the type of behavioral procedure used to investigate interval timing in rodents and primates. In addition, it should be underscored that both DA neurons and ChIs participate in the encoding of reward prediction error and they are both central to reward-guided learning. The intertwining of reward prediction and temporal processing is the source of difficulties in distinguishing neuronal signals related to expectations about future rewards from those related to timing processes (Paton \& Buonomano, 2018). This needs to be taken into account for the interpretation of the results obtained in tasks used to assess the ability of animals to time interval durations.

As emphasized above, DA and cholinergic transmissions have strong reciprocal actions on each other, but the functional outcome of these interactions and their impact on striatal output need to be further explored. There is evidence indicating that phasic changes in activity of DA neurons and ChIs tend to occur simultaneously and it has been put forward that ChI signaling would serve as a temporal frame defining the time over which the information carried by the DA signal is processed in striatal target regions (Morris et al., 2004; Cragg, 2006). Although this hypothesis has attracted much attention, further work needs to be done to understand the ways in which DA neurons and ChIs carrying temporal information act on striatal output pathways that are responsible for sending motor commands. A crucial issue for future research is to better understand the precise engagement of each system in timing behavior, for example, by using genetic tools that are capable of targeting the local cholinergic innervation of the striatum or DA midbrain projections to the striatum to assess causal effects of selectively disrupting one or the other neuronal system. 
Although DA and cholinergic transmissions have been shown to elicit changes in synaptic strength taking place within the striatal circuitry, virtually nothing is known about how these cellular mechanisms may underlie, at least in part, the processing of temporal information. One can only speculate on the role of striatal synaptic plasticity as a potential mechanism for the acquisition and maintenance of temporal memories and controlling timing behavior. Notably, in the framework of the SBF model, it has been proposed that synaptic connections are strengthened or weakened in order to produce a record in memory of learned durations (Matell \& Meck, 2004). Investigating how mechanisms of synaptic plasticity may impact performance during timing tasks are key open areas of research within the time perception field. In conclusion, the striatum continues to elicit a great deal of interest from neuroscientists as a brain region essential for the processing of temporal information. Here, we have briefly reviewed recent advances in deciphering how DA neurons and striatal ChIs may participate in the encoding of time. Future studies could aim to precisely define the key aspects of timing tasks that selectively influence the activity of these two neuronal systems. In particular, one major challenge is to disentangle neuronal activities related to timing processes from motivation, attention, and action selection, since it is well known that DA and ChI systems are involved in reward-guided learning. It is noteworthy that encoding time over ranges of seconds to minutes is required for learning to predict rewards and to guide behavior toward rewarding outcomes. In this regard, a more detailed analysis of interactions between timing and reward processing is a promising direction for future work and computational models for integrating these two aspects will also provide substantial help in clarifying the respective roles of midbrain DA neurons and striatal ChIs (Daw et al., 2006; Petter et al., 2018).

\section{Acknowledgements}


We thank the reviewers for their insightful comments and suggestions. This research was supported by Centre National de la Recherche Scientifique and the Association France Parkinson. Funding for A.C.M. was provided by a doctoral fellowship from the French government, Fondation pour la Recherche Médicale, and Fondation des Treilles.

\section{Conflict of interest}

The authors declare no competing interests.

\section{Authors contributions}

A.C.M. and P.A. wrote the manuscript.

\section{References}

Aoki, S., Liu, A. W., Zucca, A., Zucca, S. \& Wickens, J. R. (2015) Role of striatal cholinergic interneurons in set-shifting in the rat. J. Neurosci., 35, 9424-9431.

Aoki, S., Liu, A. W., Akamine, Y., Zucca, A., Zucca, S. \& Wickens, J. R. (2018) Cholinergic interneurons in the rat striatum modulate substitution of habits. Eur. J. Neurosci., 47, 1194-1205.

Apicella, P. (2002) Tonically active neurons in the primate striatum and their role in the processing of information about motivationally relevant events. Eur. J. Neurosci., 16, 20172026.

Apicella, P. (2007) Leading tonically active neurons of the striatum from reward detection to context recognition. Trends Neurosci., 30, 299-306.

Apicella, P., Legallet, E. \& Trouche, E. (1997) Responses of tonically discharging neurons in the monkey striatum to primary rewards delivered during different behavioral states. Exp. Brain Res., 116, 456-466. 
Apicella, P., Ravel, S. \& Legallet, E. (2006) A possible role for tonically active neurons of the primate striatum in learning about temporal relationships among salient stimuli. In Bezard, E. (ed), Recent Breakthroughs in Basal Ganglia Research. Nova Science Publishers, New York, pp. 55-63.

Bakhurin, K. I., Goudar, V., Shobe, J. L., Claar, L. D., Buonomano, D. V. \& Masmanidis, S. C. (2017) Differential encoding of time by prefrontal and striatal network dynamics. $J$. Neurosci., 37, 854-870.

Bell, T., Lindner, M., Langdon, A., Mullins, P. G. \& Christakou, A. (2019) Regional striatal cholinergic involvement in human behavioral flexibility. J. Neurosci., 39, 5740-5749.

Bradfield, L. A., Bertran-Gonzalez, J., Chieng, B. \& Balleine, B. W. (2013) The thalamostriatal pathway and cholinergic control of goal-directed action: interlacing new with existing learning in the striatum. Neuron, 79, 153-166.

Brown, H. D., Baker, P. M. \& Ragozzino, M. E. (2010) The parafascicular thalamic nucleus concomitantly influences behavioral flexibility and dorsomedial striatal acetylcholine output in rats. J. Neurosci., 30, 14390-14398.

Buhusi, C. V. \& Meck, W. H. (2005) What makes us tick? Functional and neural mechanisms of interval timing. Nat. Rev. Neurosci., 6, 755-765.

Buonomano, D.V. \& Karmarkar, U. R. (2002). How do we tell time? Neuroscientist, 8, $42-51$.

Cachope, R. \& Cheer, J.F. (2014) Local control of striatal dopamine release. Front. Behav. Neurosci., 8, 188.

Calabresi, P., Centonze, D., Gubellini, P., Pisani, A. \& Bernardi, G. (2000) Acetylcholine-mediated modulation of striatal function. Trends Neurosci., 23, 120 -126.

Chiba, A., Oshio, K. \& Inase, M. (2015) Neuronal representation of duration discrimination in the monkey striatum. Physiol. Rep., 3, e12283. 
Coull, J. T. \& Nobre, A. (2008) Dissociating explicit timing from temporal expectation with fMRI. Curr. Opin. Neurobiol., 18, 137-144.

Coull, J. T., Cheng, R. -K. \& Meck, W. H. (2011) Neuroanatomical and neurochemical substrates of timing. Neuropsychopharmacology, 36, 3-25.

Coull, J. T., Hwang, H. J., Leyton, M. \& Dagher, A. (2012) Dopamine precursor depletion impairs timing in healthy volunteers by attenuating activity in putamen and supplementary motor area. J. Neurosci., 32, 16704-16715.

Cragg, S. J. (2006) Meaningful silences: how dopamine listens to the ACh pause. Trends Neurosci., 29, 125-131.

Daw, N. D., Courville, A. C. \& Touretzky, D. S. (2006) Representation and timing in theories of the dopamine system. Neural Comput., 18, 1637-1677.

Davalos, D. B., Kisley, M. A. \& Ross, R. G. (2003) Effects of interval duration on temporal processing in schizophrenia. Brain and Cognition, 52, 295-301.

Di Filippo, M., Picconi, B., Tantucci, M., Ghiglieri, V., Bagetta, V., Sgobio, C., Tozzi, A., Parnettia, L. \& Calabresi, P. (2009) Short-term and long-term plasticity at corticostriatal synapses: Implications for learning and memory. Behav. Brain Res., 199, 108-118.

Fiorillo, C. D., Newsome, W. T. \& Schultz, W. (2008) The temporal precision of reward prediction in dopamine neurons. Nat. Neurosci., 11, 966-973.

Gouvêa, T. S., Monteiro, T., Motiwala, A., Soares, S., Machens, C. \& Paton, J. J. (2015) Striatal dynamics explain duration judgments. eLife, $\mathbf{4}$.

Hwang, S.-L., Gau, S. S.-F., Hsu, W.-Y. \& Wu, Y.-Y. (2010) Deficits in interval timing measured by the dual-task paradigm among children and adolescents with attentiondeficit/hyperactivity disorder. J. Child Psychol. Psychiatry, 51, 223-232.

Hollerman, J. R. \& Schultz, W. (1998) Dopamine neurons report an error in the temporal prediction of reward during learning. Nat. Neurosci., 1, 304-309. 
Jin, D. Z., Fujii, N. \& Graybiel, A. M. (2009) Neural representation of time in corticobasal ganglia circuits. Proc. Natl. Acad. Sci. USA, 106, 19156-19161.

Kataoka, Y., Kalanithi, P. S. A., Grantz, H., Schwartz, M. L., Saper, C., Leckman, J. F. \& Vaccarino, F. M. (2010) Decreased number of parvalbumin and cholinergic interneurons in the striatum of individuals with Tourette syndrome. J. Comp. Neurol., 518, 277-291.

Kobayashi, S. \& Schultz, W. (2008) Influence of reward delays on responses of dopamine neurons. J. Neurosci., 28, 7837-7846.

Matell, M.S.\& Meck, W.H.(2000) Neuropsychological mechanisms of interval timing behavior. Bioessays, 22, 94-103.

Matell, M. S., Meck, W. H. \& Nicolelis, M. A. L. (2003) Interval timing and the encoding of signal duration by ensembles of cortical and striatal neurons. Behav. Neurosci., $117,760-773$

Matell, M. S., King, G. R. \& Meck, W. H. (2004) Differential modulation of clock speed by the administration of intermittent versus continuous cocaine. Behav. Neurosci., 118, 150156.

Meck, W. H. (1983) Selective adjustment of the speed of internal clock and memory processes. J. Exp. Psychol. Anim. Behav. Process., 9, 171-201.

Meck, W. H. (1996) Neuropharmacology of timing and time perception. Brain Res. Cogn. Brain Res., 3, 227-242.

Meck, W. H. (2002) Choline uptake in the frontal cortex is proportional to the absolute error of a temporal memory translation constant in mature and aged rats. Learn. Motiv., 33, 88104.

Meck, W. H. \& Church, R. M. (1987) Cholinergic modulation of the content of temporal memory. Behav. Neurosci., 101, 457-464. 
Mello, G. B. M., Soares, S. \& Paton, J. J. (2015) A scalable population code for time in the striatum. Curr. Biol., 25, 1113-1122.

Merchant, H., Luciana, M., Hooper, C., Majestic, S. \& Tuite, P. (2008) Interval timing and Parkinson's disease: Heterogeneity in temporal performance. Exp. Brain Res., 184, 233 248.

Merchant, H., Harrington, D. L. \& Meck, W. H. (2013) Neural basis of the perception and estimation of time. Annu. Rev. Neurosci., 8, 313-36.

Mioni, G., Capizzi, M., Vallesi, A., Correa, Á., Di Giacopo, R. \& Stablum, F. (2018) Dissociating explicit and implicit timing in Parkinson's disease patients: evidence from bisection and foreperiod tasks. Front. Hum. Neurosci., 12, 17.

Morris, G., Arkadir, D., Nevet, A., Vaadia, E. \& Bergman, H. (2004), Coincident but distinct messages of midbrain dopamine and striatal tonically active neurons. Neuron, 43, 133143.

Okada, K., Nishizawa, K., Fukabori, R., Kai, N., Shiota, A., Ueda, M., Tsutsui, Y., Sakata, S., Matsushita, N. \& Kobayashi, K. (2014) Enhanced flexibility of place discrimination learning by targeting striatal cholinergic interneurons. Nat. Commun., 5, 3778.

Oprisan, S. A. \& Buhusi, C. V. (2011) Modeling pharmacological clock and memory patterns of interval timing in a striatal beat-frequency model with realistic, noisy neurons. Front. Integr. Neurosci., 5, 52.

Parker, K. L., Lamichhane, D., Caetano, M. S. \& Narayanan, N. S. (2013) Executive dysfunction in Parkinson's disease and timing deficits. Front. Integr. Neurosci., 7, 75.

Pasquereau, B \& Turner, R. S. (2015) Limited encoding of effort by dopamine neurons in a cost-benefit trade-off task. $J$. Neurosci., 33, 8288-8300.

Pastor, M. A., Artieda, J., Jahanshahi, M. \& Obeso, J. A. (1992) Time estimation and reproduction is abnormal in Parkinson's disease. Brain, 115, 211-225. 
Paton, J. J. \& Buonomano, D. V. (2018) The neural basis of timing: distributed mechanisms for diverse functions. Neuron, 98, 687-705.

Petter E. A., Gershman S. J. \& Meck, W. H. (2018) Integrating Models of Interval Timing and Reinforcement Learning. Trends Cogn. Sci., 22, 911-922.

Pisani, A., Bernardi, G., Ding, J. \& Surmeier, D. J. (2007) Re-emergence of striatal cholinergic interneurons in movement disorders. Trends Neurosci., 30, 545-553.

Prado, V.F., Janickova, H., Al-Onaizi, M.A. \& Prado, M.A. (2017) Cholinergic circuits in cognitive flexibility. Neuroscience, $\mathbf{3 4 5}, 130-141$.

Rammsayer, T. (1990) Temporal discrimination in schizophrenic and affective disorders: Evidence for a dopamine-dependent internal clock. Int. J. Neurosci., 53, 111-120.

Ravel, S., Sardo, P., Legallet, E. \& Apicella, P. (2001) Reward unpredictability in- side and outside of a task context as a determinant of the responses of tonically active neurons in the monkey striatum. J. Neurosci., 21, 5730- 5739.

Sardo, P., Ravel, S., Legallet, E. \& Apicella, P. (2000) Influence of the predicted time of stimuli eliciting movements on responses of tonically active neurons in the monkey striatum. Eur. J. Neurosci., 12, 1801-1816.

Smith, A., Cubillo, A., Barrett, N., Giampietro, V., Simmons, A., Brammer, M. \& Rubia, K. (2013) Neurofunctional effects of methylphenidate and atomoxetine in boys with attention-deficit/hyperactivity disorder during time discrimination. Biol. Psychiatry, 74, 615622.

Soares, S., Atallah, B. V. \& Paton, J. J. (2016) Midbrain dopamine neurons control judgment of time. Science, 354, 1273-1277.

Stalnaker, T.A., Berg, B., Aujla, N. \& Schoenbaum, G. (2016) Cholinergic interneurons use orbitofrontal input to track beliefs about current state. J. Neurosci., 36, 6242-6257. 
Threlfell, S., Lalic, T., Platt, N. J., Jennings, K. A., Deisseroth, K. \& Cragg, S. J. (2012) Striatal dopamine release is triggered by synchronized activity in cholinergic interneurons. Neuron, 75, 58-64.

Vicario, C. M., Martino, D., Spata, F., Defazio, G., Giacche, R., Martino, V., Rappo, G., Pepi, A. M., Silvestri, P. R. \& Cardona, F. (2010) Time processing in children with Tourette's syndrome. Brain Cogn., 73, 28-34.

Volz, H. P., Nenadic, I., Gaser, C., Rammsayer, T., Häger, F. \& Sauer, H. (2001) Time estimation in schizophrenia: An fMRI study at adjusted levels of difficulty. Neuroreport, 12, $313-316$.

Wang, J., Narain, D., Hosseini, E. A. \& Jazayeri, M. (2018) Flexible timing by temporal scaling of cortical responses. Nat. Neurosci., 21, 102-110.

Yang, B., Chan, R. C. K., Zou, X., Jing, J., Mai, J. \& Li, J. (2007) Time perception deficit in children with ADHD. Brain Res., 1170, 90-96.

Yc, K., Prado, L. \& Merchant, H. (2019) The scalar property during isochronous tapping is disrupted by a D2-like agonist in the nonhuman primate. J. Neurophysio1., 21, 940-949.

Ztaou, S., Maurice, N., Camon, J., Guiraudie-Capraz, G., Kerkerian-Le Goff, L., Beurrier, C., Liberge, M. \& Amalric, M. (2016) Involvement of Striatal Cholinergic Interneurons and M1 and M4 Muscarinic Receptors in Motor Symptoms of Parkinson's Disease. J. Neurosci., 36, 9161-9172. 


\section{Figure legends}

Figure 1. Modulation of dopamine neuron and cholinergic interneuron activity when reward occurs at a different time as predicted. Monkeys were trained on an instrumental task (A) or a Pavlovian conditioning task $(\mathbf{B})$ in which a lever contact or a visual stimulus is followed, after a constant time interval (1s), by the delivery of a liquid reward. During these experiments, the interval was occasionally shortened or lengthened across successive trial blocks. Each line of dots indicates the neuronal activity during a single trial aligned on the onset of the lever contact or visual stimulus. The sequence of trials is shown chronologically from top to bottom in each block. Both neurons did not change their activity when reward was delivered at the end of the accustomed 1-s delay, whereas they displayed a brief increase (A) or decrease (B) in activity when reward was delivered earlier or later than the usual time, suggesting a sensitivity to temporal aspects of reward prediction. The DA neuron also showed a decrease in activity at the habitual time of the reward when it was delayed beyond the expected time, reflecting the known role of these neurons in reward prediction error signaling (i.e., the difference between expected and received reward). The cholinergic TAN also responded to the visual stimulus predictive of reward, and this response was unchanged across the different trial blocks. (A) modified from Hollerman and Schultz 1998; (B) modified from Apicella 2017.

Figure 2. Modulation of dopamine neuron activity during a temporal discrimination task. A. Task event sequence. Mice were trained to keep track of the time elapsed between two auditory stimuli in order to make decisions as to whether the intertone interval was shorter or longer than a pre-learned 1.5-s interval duration. Animals' judgements about the duration of time intervals (i.e., shorter or longer) were expressed as movements toward one or the other side. The grayshaded area indicates the interval reference. B. Photometric recordings of DA neuron activity during task performance. Trials were divided into three groups (High, Med, and Low) according 
to the strength of the DA response to the second tone for a given interval (1.74 second). C. Psychometric curves corresponding to trials from each group. The magnitude of the DA response increased when mice judged the duration of time intervals as being shorter than the memorized standard (i.e., underestimation). The inset shows the difference in the probability of making a long choice between Med and Low or High (red or blue) DA responses. Adapted, with permission, from Soares et al. 2016.

Figure 3. Modulation of cholinergic TAN activity during two timing task. A. Sequence of task events. Left, In the duration estimation task, the monkey kept the hand resting on a bar and a visual stimulus on the left or the right side marked the beginning of a particular time interval (short or long, in the seconds range) with the cue location indicating interval duration. The monkey had to wait until the time interval has elapsed before reaching the target on the side of the stimulus. Right, In the Pavlovian protocol, the presentation of a visual stimulus was automatically followed by reward at the end of the same two time intervals as in the duration estimation task. The areas shaded gray represent the minimum waiting time before self-initiated movements (left) and the duration of the stimulus-reward interval (right). B. Populationaveraged responses of TANs to the timing cue in the duration estimation task, separately for short and long intervals. The vertical dotted line indicates the cue onset. n, number of neurons. C. Influence of accuracy of duration estimation on the magnitude of the TAN response. Trials were divided into different groups according to movement onset time and magnitudes of change were calculated to include only the trials within a specific range, separately for short and long intervals. Movements starting before the movement onset threshold (-500/0 range) were not rewarded. The magnitude of the pause response did not differ among different trial ranges in the population of recorded TANs. D. Population-averaged responses of TANs to the timing cue in the Pavlovian protocol, separately for short and long intervals. The vertical dotted line 
indicates the onset of the reward-predicting stimulus. n, number of neurons. E. Selectivity of TAN responses to the timing cue for a particular condition. The percentages are calculated from the total number of TANs recorded in both the duration estimation task and the Pavlovian conditioning tasks. $\mathrm{n}$, number of neurons. DET, duration estimation task; PCT, Pavlovian conditioning task. Martel and Apicella, unpublished results. 
Figure 1

A midbrain dopamine neuron

1-s interval
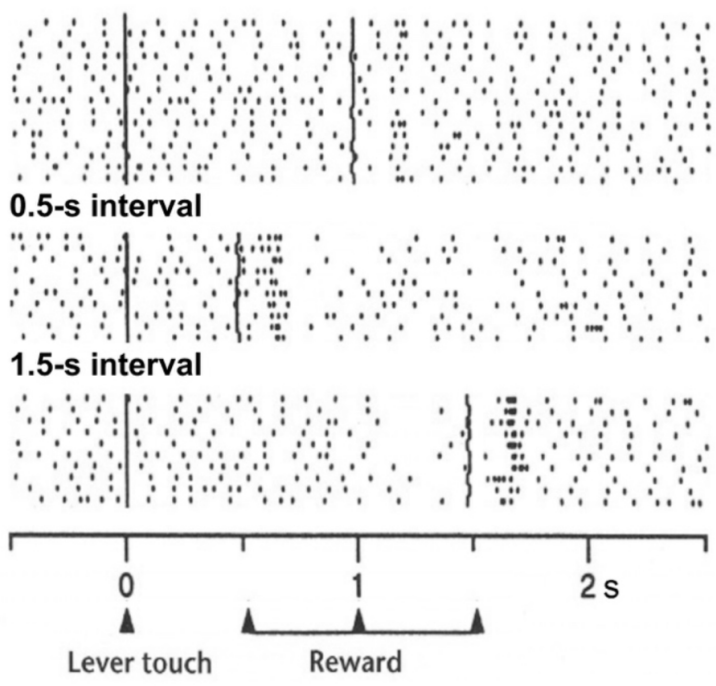

B striatal cholinergic interneuron
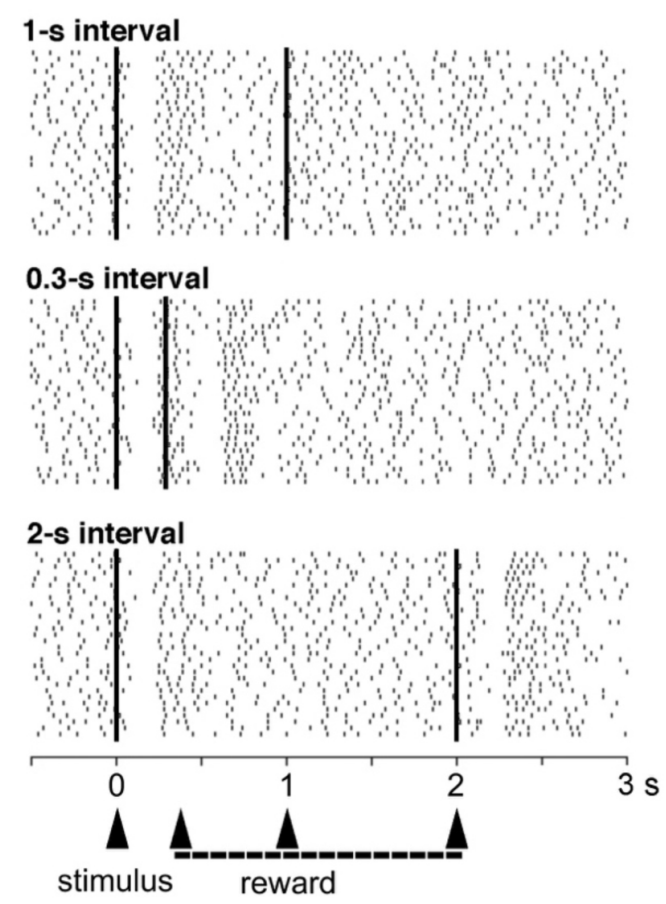
Figure 2
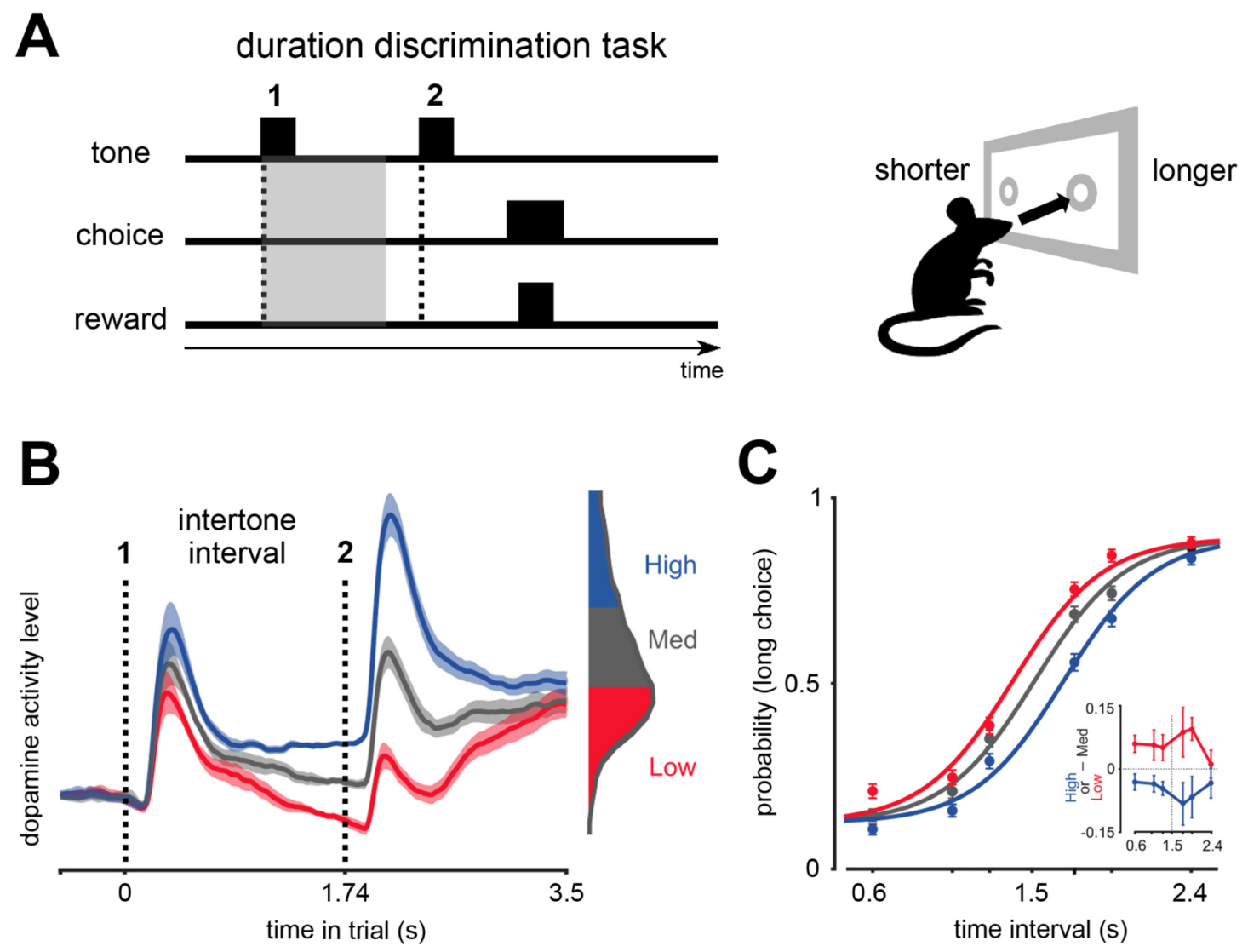
Figure 3

A
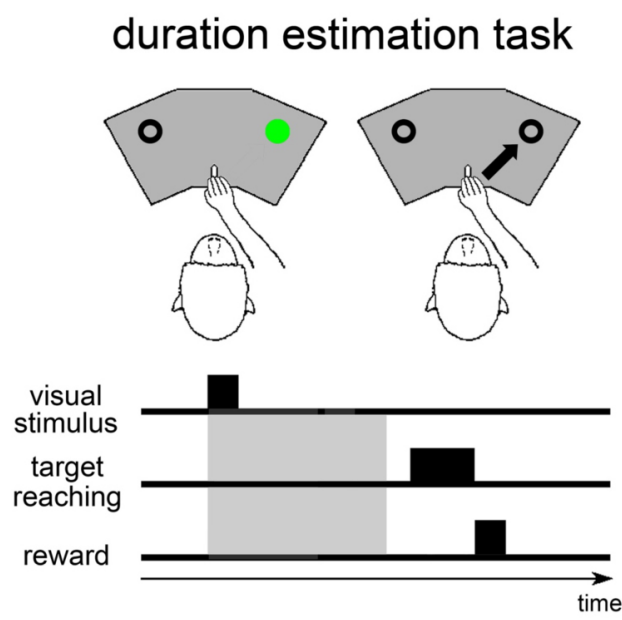

B duration estimation task

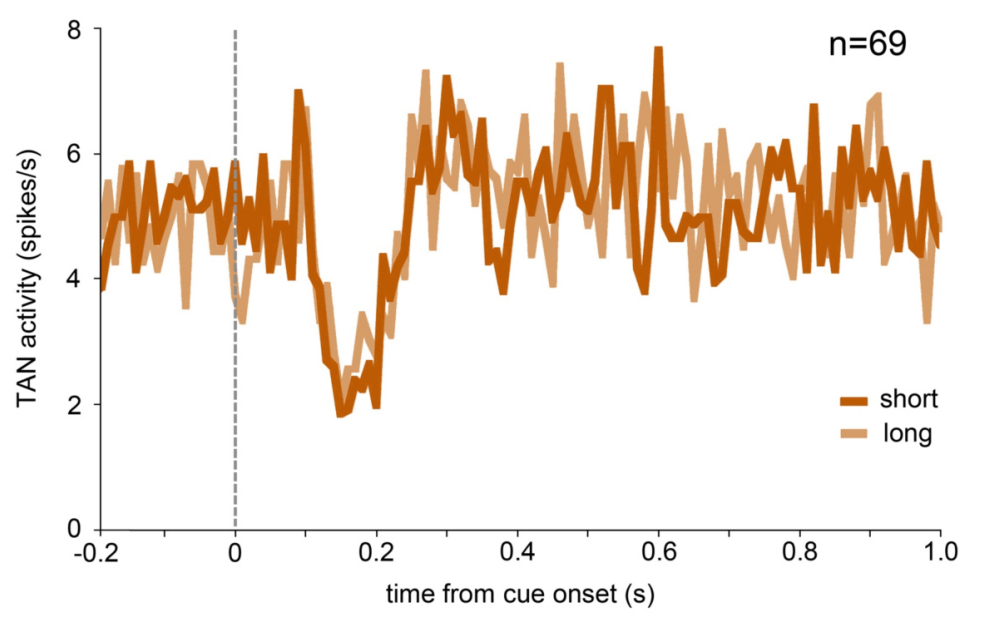

D pavlovian conditioning task

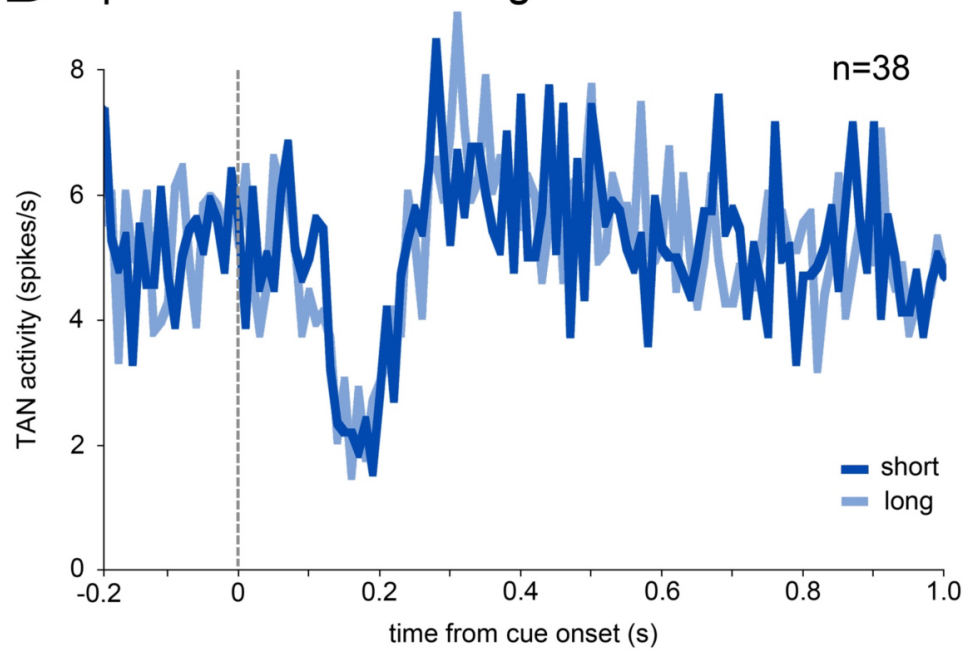

pavlovian conditioning task
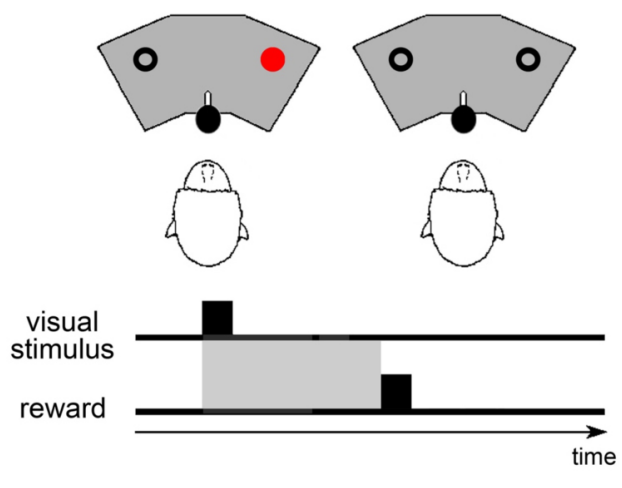

C

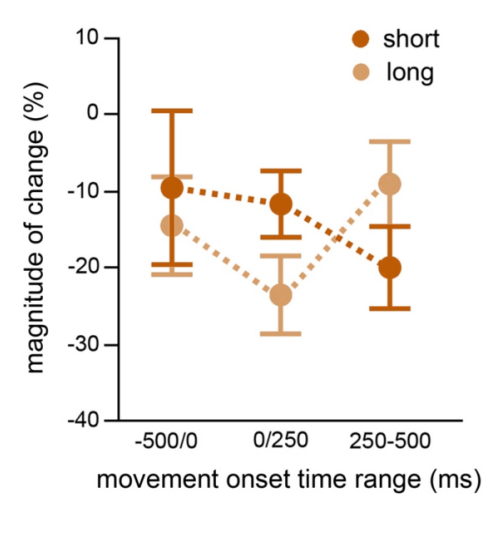

E

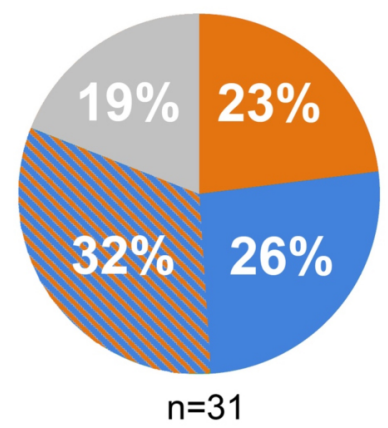

responsive only in DET

responsive only in $\mathrm{PCT}$

responsive in DET and $\mathrm{PCT}$ unresponsive 